\title{
Massive hemoptysis due to bronchopulmonary arterial fistula successfuly treated with amplatzer plug occlusion
}

\author{
Monzer A. Chehab ${ }^{1}$ Sivateja Mandava², Richard Smillie ${ }^{1}$, Adam H. Lackey ${ }^{3}$, Hugh Chung ${ }^{3}$, \\ Stephen A. Vartanian'
}

1. Department of Radiology, Beaumont Health System, Royal Oak, United States. 2. Oakland University William Beaumont School of Medicine, Rochester, United States. 3. Department of Thoracic Surgery, Beaumont Health System, Royal Oak, United States.

Correspondence: Sivateja Mandava. Address: Oakland University William Beaumont School of Medicine, Rochester, MI, United States. E-mail: smandava@oakland.edu

Received: September 28, 2014

DOI : $10.5430 /$ jbgc.v4n4p68
Accepted: November 4, 2014

URL: http://dx.doi.org/10.5430/jbgc.v4n4p68

\section{Abstract}

We present the case of a 59-year-old male with massive hemoptysis secondary to a bronchopulmonary artery fistula formed as the result of a perihilar lung cancer. The hemoptysis was successfully treated by Amplatzer Plug (St. Jude Medical) embolization of the supplying pulmonary artery branch. At the same time, bronchoscopy was performed to tamponade the patients bleeding and avoid exsanguination. Biopsy of the mass demonstrated poorly differentiated non small cell carcinoma. Post procedure day 13 the patient underwent staging with PET CT which showed localized disease. His hemoptysis resolved.

\section{Key words}

Bronchial-pulmonary artery fistula, Amplatzer plug, Bronchoscopy assisted, Hemoptysis

\section{Introduction}

A Bronchopulmonary Artery Fistula (BPAF) is an exceedingly rare entity that is limited to a handful of case reports in the literature. A fistula is formed when there is erosion of one lumenal compartment into another. In the case of BPAF, there is a communicating channel between the pulmonary artery and the bronchial tree. Such a connection would allow for the free passage of blood from the artery into the bronchial tree resulting in hemoptysis, impaired gas exchange, and hemodynamic instability. Given the limited data, there is no consensus yet on the optimal way to treat this condition, nor has there been a reliable way to diagnose this condition. Multiple options for treatment have been explored, some as drastic as removing the entire lobe of lung that is affected. Below we present a case of a BPAF in a 59-year-old male who presented to our facility with massive hemoptysis, successfully treated using an Amplatzer Vascular Plug.

\section{Case report}

A 59-year-old male with a greater than 30 pack year smoking history was transferred from an outside facility after expectorating $\sim 1$ pint of blood. The patient reported a progressively worsening dry cough over the three months and $30 \mathrm{lb}$ 
weight loss over the past year. He denied any history of malignancy or chemoradiation therapy. Contrast enhanced chest CT demonstrated a right perihilar mass with encasement and narrowing of the bronchus intermedius and descending branch of the right pulmonary artery (see Figure 1a and 1b). The following day, bronchoscopy demonstrated a fungating mass intruding into the bronchus intermedius with stigmata of recent bleeding. Following bronchoscopy, biopsy and debulking, hemostasis was achieved with endobronchial cautery, APC as well as application of topical 1:20,000 epinephrine. Six days following admission, the patient had an acute episode of massive dark red hemoptysis, with oxygen desaturation to $75 \%$. His hemoglobin measured $6.8 \mathrm{~g} / \mathrm{dl}$ which had decreased from presentation of $9.4 \mathrm{~g} / \mathrm{dl}$. He was intubated and taken for repeat bronchoscopy which demonstrated active, dark red bleeding with the bronchus intermedius (see Figure 1c). Interventional Radiology was urgently consulted to help localize the site of bleeding.

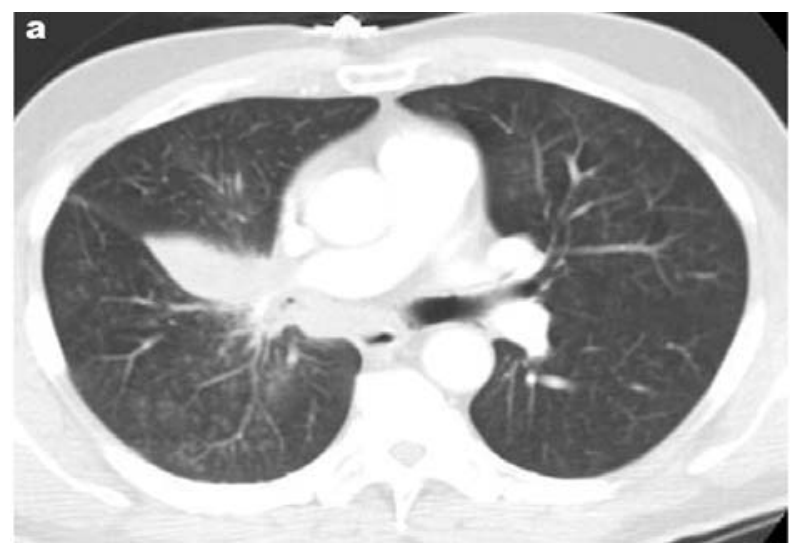

Figure 1a. 59 year old male with bronchial-pulmonary artery fistula. Axial contrast enhanced CT with right upper lobe mass infiltrating the right bronchus intermedius.

Figure 1b. Contrast enhanced CT with a mass along the medial minor fissure encasing and narrowing the lumen of the right main pulmonary artery.

Figure 1c. Diagnostic bronchoscopy demonstrates fungating mass eroding into the bronchus intermedius.

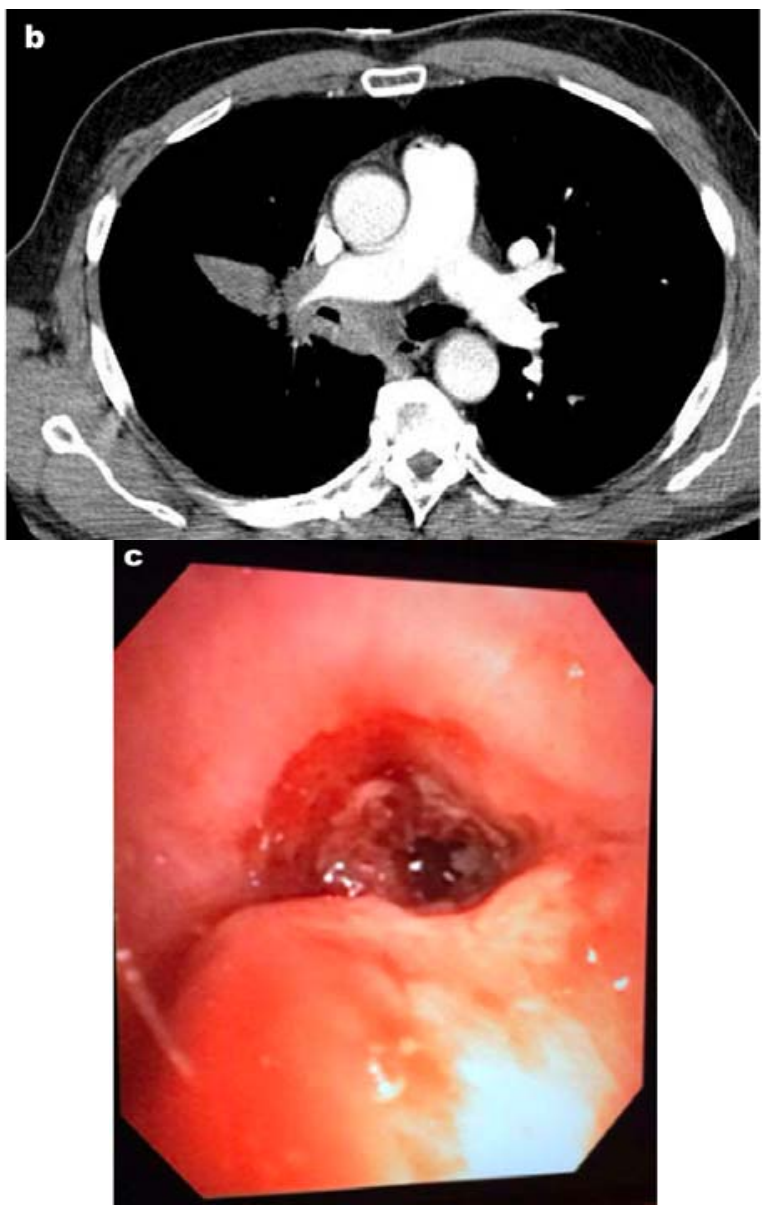

The patient was urgently taken for angiography. Working through a 5 French right common femoral artery vascular sheath, a 5 French Michelson Catheter, Simmons II catheter and pigtail catheter did not identify a bronchial arterial bleed (see Figure 2a). No enlarged bronchial arteries were identified. In fact, the bronchial arteries were somewhat small in appearance and were technically challenging to access. Because of the dark nature of the bleeding, pulmonary arteriography was performed via an APC Davis Catheter (Cook ${ }^{\circledR}$, Bloomington Indiana). This demonstrated a stricture of the proximal aspect of the descending branch of the right pulmonary artery (see Figure 2b) without evidence of active extravasation. Access distal to the stenosis was carefully gained following exchange for a Berenstein catheter (Angiodynamics ${ }^{\circledR}$, Latham, New York) over an 035 Glidewire (Terumo ${ }^{\circledR}$, Tokyo Japan). During this, the distal tip of catheter was noted to enter a non-vascular structure. A small amount of Isovue $370^{\circledR}$ (Bracco, Milan Italy) iodinated contrast was instilled outlining the right lower lobe bronchus and its distal branches (see Figure 2c). With continued bleeding noted through the endotracheal tube and concerns about the patients hemodynamic status. It was felt that removing the catheter from the fistula may "remove the plug" and flood the bronchus with pulmonary venous blood. 
Thoracic surgery then urgently performed bronchoscopy. Using a 10 French flexible bronchoscope, a 22 French balloon was inflated, occluding the right lower lobe bronchus (see Figure 3a). At this point, urgent therapeutic options were reviewed. Pneumonectomy was felt to carry a high mortality rate in this acute setting. Placement of a covered stent was also felt to be inappropriate given the invasive nature of the eroding lesion. In addition, a covered stent was not used in this case because of difficulty gaining distal access in the pulmonary artery beyond the fistula. The decision was then made to occlude the right lower lobar pulmonary artery. The catheter was exchanged for a 6 French Rabbe Catheter (Cook ${ }^{\circledR}$ Bloomington Indiana) through which a $12 \mathrm{~mm}$ Amplatzer Vascular Plug (St. Jude Medical, St. Paul Minnesota) was deployed, occluding right lower lobe pulmonary artery (see Figure 3b). Post embolization angiogram demonstrates absence of flow distal to the plug (see Figure 3c). The occlusion balloon was deflated and immediately, the hemoptysis ceased.
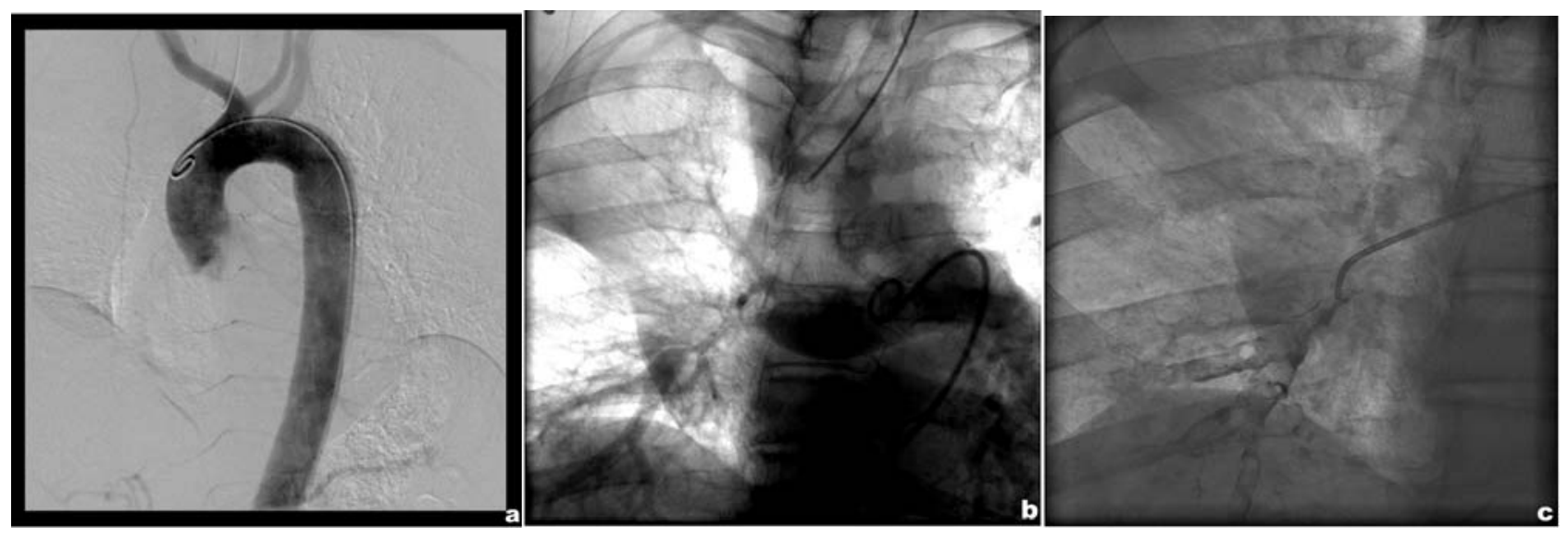

Figure 2. a. Aortogram demonstrates no enlarged bronchial arteries. Multiple attempts to cannulate the bronchial arteries were made. b. Pulmonary arteriogram with focal narrowing of the descending branch of the right pulmonary artery. c. Fluoroscopic spot image demonstrates distal tip of a catheter placed through the right pulmonary artery whose distal tip entering bronchus intermedius through fistulous communication. Contrast opacification demonstrated outline of the right lower lobe bronchi.

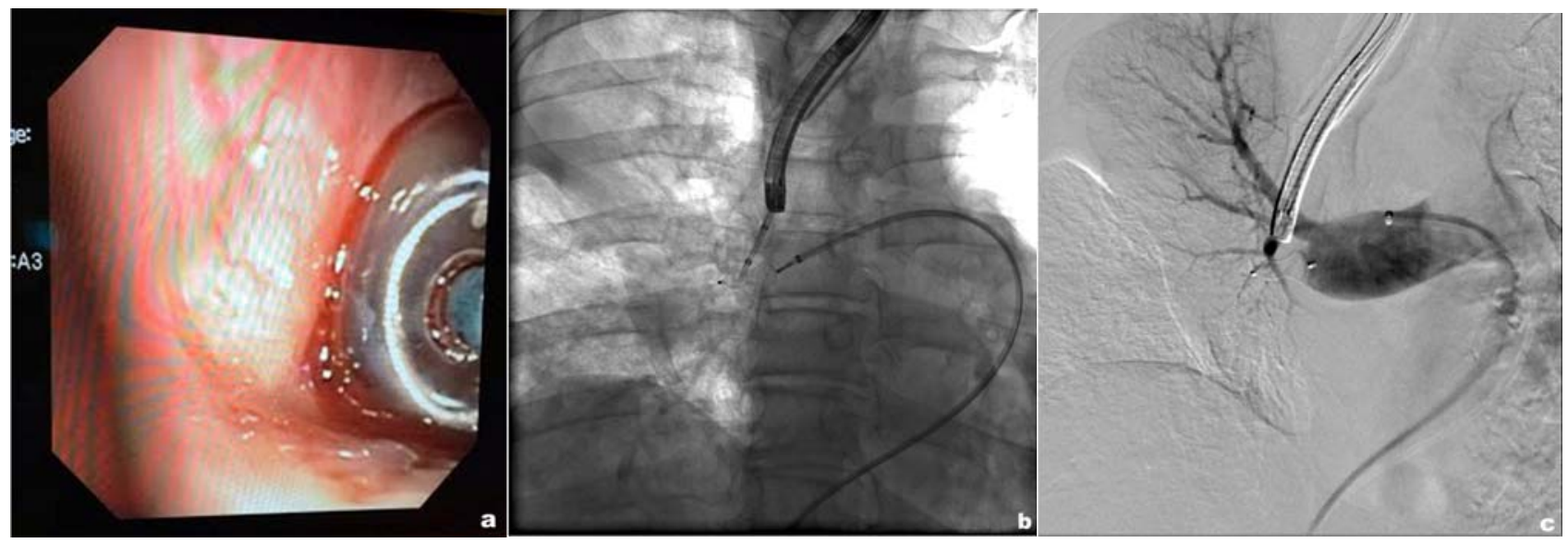

Figure 3. a. Intraoperative bronchoscopy during balloon inflation to tamponade bleeding. b. Fluoroscopic spot image obtained immediately following deployment of a $12 \mathrm{~mm}$ Amplatzer plug into the descending branch of the right lower lobe pulmonary artery. c. Digital subtraction angiogram following Amplatzer plug deployment demonstrating complete occlusion of the right lower lobe pulmonary artery.

The patient was extubated safely the following day with stable hemodynamics. He did have a cough for the first few days without significant bleeding. No further blood transfusions were necessary. On post procedure day 4 . The patient 
underwent PET CT which demonstrated multiple FDG avid mediastinal and axillary lymph nodes. The patient was discharged in stable condition with plans to pursue external beam radiation and chemotherapy.

\section{Discussion}

Bronchopulmonary artery fistula refers to an abnormal communication between a pulmonary artery branch and a bronchus. The condition is exceedingly rare, its occurrence restricted to case reports ${ }^{[1]}$. The condition has been described as a complication of an inflammatory process in the presence of Aspergillus infection ${ }^{[2]}$, lung resection ${ }^{[3]}$, lung transplantation ${ }^{[4]}$, Swan-Ganz catheter placement ${ }^{[5]}$ and ulcerative bronchitis ${ }^{[6]}$. Cases of malignancy as the underlying mechanism have been reported, likely complicated by prior radiation therapy ${ }^{[7]}$. In our case, the fistula was the result of abnormal channel formation secondary to an invasive neoplasm without a prior history of radiation.

The rarity of the condition and complexity of underlying pathology makes its recognition difficult on non-invasive imaging. The fistula may be hidden from view as a result of simultaneously existing consolidation due to atelectasis ${ }^{[6]}$ or ongoing infection such as Aspergillosis ${ }^{[2]}$. CT Angiography has been described as useful in post lobectomy patients as possibly showing a residual space containing air-fluid level as well as bubbles around the bronchial stump which is highly suspicious for BPAF ${ }^{[8]}$. Bronchoscopy may also provide valuable diagnostic information if it shows a dark venous blood within the endobronchialtree ${ }^{[9]}$.

In the literature, a variety of treatment options have been described largely depending on the underlying etiology. Smaller lesions may undergo careful monitoring with bronchoscopy and local hemostasis techniques. Surgical options such as lobectomy carry a significant risk of mortality (up to $27 \%$ ), especially in the acute setting ${ }^{[10]}$. Self expanding covered stents offer an alternative option which may be useful in preserving blood flow to the artery while avoiding a large surgical procedure ${ }^{[8]}$. In our case, a covered stent was not felt to be feasible given the significant narrowing of the pulmonary artery, unlikely to accommodate the 9-10 French sheath used to deliver the covered stent. Furthermore, the fungating mass would likely further encase the stent and erode around it. Complete occlusion of supplying vessel was felt to be appropriate proximal to the fungating mass. This was done without fear of parenchymal necrosis provided the dual blood supply of the lung. The use of an Amplatzer plug offered a safe and rapid treatment for life threatening hemoptysis through a minimally invasive technique.

\section{Conclusion}

In the event of massive hemoptysis, plug embolization of the artery feeding a bronchopulmonary artery fistula provides a rapid, safe and effective treatment. In our case, it provided a life saving temporization such that the patient could undergo further therapy.

\section{References}

[1] Conlan AA, Hurwitz SS, Krige L, Nico-laou N, Pool R. Massive hemoptysis: review of 123 cases. J Thorac Cardiovasc Surg. 1983; 85: 120-12. PMid: 6848880.

[2] Christopher G, Slatore MD. Bronchial-Pulmonary Artery Fistula With Fatal Massive Hemoptysis Caused by Anastomotic Bronchial Aspergillus Infection in a Lung Transplant Recipient RESPIRATORY CARE: NOVEMBER. 2007; 52(11).

[3] Forcillo J, Liberman M, Gorgos A, Ferraro P. Repair of a bronchovascular fistula four years after right carinal pneumonectomy. Ann Thorac Surg. 2013; 95(6): 2152-2153. PMid: 23706434. http://dx.doi.org/10.1016/j.athoracsur.2012.10.075

[4] Birsan T, Taghavi S, Klepetko W. Bronchial-pulmonary artery fistula after unilateral lung transplantation: a case report. J Heart Lung Transplant. 1998; 17: 437-438. PMid: 9588590.

[5] Rubin SA, Puckett RP. Pulmonary artery--bronchial fistula: A new complication of swan-ganz catheterization. Chest. 1979; 75(4): 515-516. http://dx.doi.org/10.1378/chest.75.4.515

Published by Sciedu Press 
[6] Abe J., Kanma K., Saito, Y. Pulmonary artery bronchial fistula after sleeve lobectomy: A case report. The internet journal of pulmonary medicine. 2000; 2(1).

[7] Ruffini E, Oliaro A, Mancuso M, et al. Successful management of hemorrhage from the pulmonary artery stump after lobectomy for bronchogenic carcinoma. J CardiovascSurg (Torino). 1994; 35: 257-259.

[8] Davison BD, Ring DH, Bueno R, Jaklitsch MT. Endovascular stent-graft repair of a pulmonary artery-bronchial fistula. J Vasc Interv Radiol. 2003; 14(7): 929-932. PMid: 12847202. http://dx.doi.org/10.1097/01.RVI.0000082825.75926.82

[9] Pinet C, Palka W, Metras D, Thomas P, Meric B, Dumon JF. Management of an intrabronchial rupture of right main pulmonary artery: A case report. Chest. 2002; 121(3): 988-990. PMid: 11888988. http://dx.doi.org/10.1378/chest.121.3.988

[10] Jougon J, Ballester M, Delcambre F, et al. Massive hemoptysis: what place for medical and surgical treatment. Eur J Cardiothorac Surg. 2002; 22: 345-351. http://dx.doi.org/10.1016/S1010-7940(02)00337-8 